\title{
Checklist of Palatable Grass Species from Peninsular India
}

\author{
Puroshottam Dattatraya GORADE, Mandar Nilkanth DATAR* \\ Plant Science Division, Agharkar Research Institute, Maharashtra, India; mndatar@aripune.org, \\ puroshottam.gorade@gmail.com (*orrespondingauthor)
}

\begin{abstract}
Grasslands are common landscapes of India and dominate peninsular Indian landmass. Though secondary, these grasslands are important from cultural as well as economical point of view. Domestic cattle population of this region largely depends on these grasslands as food source. The grasslands, exposed to anthropocentric activities like burning and grazing, are facing several changes like replacement of palatable species by unpalatable ones. As an attempt to understand the fodder potential of grasslands of this region, a comprehensive checklist of palatable grass species, based on literature survey and field experience, was compiled. Local pastoral communities were interviewed for information on palatable grass species and their utility potentials. Various herbaria were consulted for confirmation of habitats, presence and absence of awns and grass phenology. The grasses with awns are consumed before the maturation and after the dispersal of awns. A total of 143 grass species were documented as palatable, of which 64 are awned and 79 are unawned. The palatable grasses were classified in 9 habitats and the palatability grade based on their use value is assessed. This documentation will be helpful for understanding and better management of grasslands of peninsular India.
\end{abstract}

Keywords: fodder potential, grasslands, grasses, habitats

\section{Introduction}

Grasslands are grounds covered by vegetation dominated by grasses with little or no tree cover (Suttie et al., 2005). Grasslands are important landscape due to their ability to provide fodder for livestock and wild animals. In grasslands, grasses form bulk of the fodder though they are not exclusively consumed by cattle, but legumes, other forbs and sedges are also consumed. Grasses are an advanced group of monocot plants that produce high quantity of biomass in short life cycle, and by this they play a vital role in food, fodder and economy of the world. Their high production of biomass is the result of a special photosynthetic pathway known as $\mathrm{C} 4$ adapted by majority of the grasses in addition to normal photosynthesis mode. Grasslands are natural source of fodder for domestic animals and occupy about $31-43 \%$ of the total surface of the Earth, about $20 \%$ of Asia and more than $10 \%$ of India (Pemadasa, 1990). Grasslands are often treated as wasteland and are converted to agricultural or industrial areas. Very few grasslands in the world have remained without any human interference. Human interference has resulted in changes in overall ecology of these habitats. Palatable grasses are slowly replaced by unpalatable grasses because of heavy grazing by livestock (Moretto and Distel, 1991; Young and Solbrig, 1993).

Indian grasslands are degraded stage of deciduous forests and hence are not climax grasslands (Champion and Seth, 1968). In India, with a wide range of environmental variables, the climax can be either forest or desert and the grasslands that exist in the country are in seral stage (Bor, 1960). These grasslands are spread all over the country, but dominate the Peninsular Indian landmass.

Peninsular India is extending beyond the bed of Ganga river across the Indo-Gangetic plains of North India. The Indo-Gangetic plains represent the boundary of Peninsula. In peninsular India natural habitats are destructed by man within historical time, for the reason that the HumidTropical forests have been transformed into semi-arid deciduous forest or even into scrubland and savanna (Mani, 1974). Maharashtra, an important state in Peninsular India, has a long coast along Arabian Sea which accounts for $9.36 \%$ of the country's coastal area. The region lies between $22^{\circ} 1^{\prime}$ to $16^{\circ} 4^{\prime}$ north latitude and $72^{\circ} 6^{\prime}$ to $80^{\circ} 1^{\prime}$ east longitude with an area of 307,690 sq. $\mathrm{km}$. (Lakshminarsimhan, 1996; Potdar et al., 2012). In Maharashtra grasses are represented by 125 genera, 415 species, 2 subspecies and 41 varieties. All of these species are spread across all the habitats, while only a limited number of species can be called as true grassland grasses. The grassland community in Maharashtra grasslands is described as Dichanthium-Sebima type on the basis of dominant vegetation of grass species (Dabadghao and Shankarnarayan, 1973). On the basis of soil depth, topography and hydrography, grasslands of Maharashtra are classified in seven major and twenty four minor patterns (Oke, 1973). There are many pastoral communities in peninsular India which directly depend on grassland habitats. Gavli dhangar, one of the pastoral communities depending on grasslands, is 
442

primarily buffalo keeping. In addition to buffalo, they keep other cattle, such as sheep and goats. This community has knowledge about grass species preferred by domestic animals and vernacular names (Gadgil and Malhotra, 1982). Traditionally some grassland patches are protected by local people, called as Kuran or Gairan and are used as a good source of palatable grass. Most of these protected patches occur in the form of Grassland-cultivation mosaic. One third of the fodder requirement for livestock comes from cultivation, while for the rest grasslands are the only source (Gadgil, 1993). Grasslands in this region are either exposed to cattle grazing or the grass is being harvested and fed to cattle. Burning is another activity that is often practiced in Peninsular Indian grasslands. This is done by local people with a point of view that after burning the grassland would facilitate growth of new grasses through seed germination in the next season (Suttie et al., 2005). But these activities have resulted in palatable grasses being slowly replaced by unpalatable grasses (Naik and Patunkar, 1979). To augment palatable species in grasslands is the biggest challenge in front of grassland managers of this region.

Palatability is consumption of plant or plant parts with relish by grazing animal (Husain and Durrani, 2009). Generally palatability and preference are used as synonymous, though preference is essentially behavioral, which is totally depending on the choice of the grazing animals (Ivins, 1952). The palatability of the grass is dependent on the chemical constituents and nutritional content such as carbohydrates, proteins, fiber etc. and their proportions, which are regulated by environmental factors like topography, climate etc. (Jawed et al., 2008). At the maturity of the grass, protein content decreases, while fiber, lignin, cellulose etc. increases, hence grasses are more acceptable when they are young (Heady, 1964; Mirza et al., 2002). Some morphological modifications such as awns, leaf margins produced by grasses also affect the palatability of the particular grass species as these modifications cause injuries to oral cavities of grazing animals, so that by these modifications the grasses are avoided by cattle.

The present work, fodder potential of Peninsular Indian grasslands is understood by assessing palatability of various species of grasses growing in various habitats in addition to grasslands. A comprehensive checklist of palatable grasses of Peninsular India is provided here.

\section{Materials and methods}

A preliminary checklist for palatable grasses from Maharashtra region of Peninsular India based on detail information about flowering season, habitats, vernacular names of grass species and palatability status was compiled (Blatter and McCann, 1935; Bor, 1960; Patunkar, 1980; Kulkarni, 1995; Potdar, 2012). For confirmation of this checklist local livestock holders and people were interviewed from different regions for preferred grass species by domestic animals. Vernacular names, both from literature and those used by pastoral communities, were attributed to the species wherever possible. These names are very region specific and people from different region use different vernacular names for same grass. Specimens deposited in herbaria, such as Botanical Survey of India, Western Regional Center (BSI) and Agharkar Research Institute Herbarium (AHMA) were consulted for parameters such as flowering season, presence or absence of awns. Online World Grass Flora available at Royal Botanic Garden, Kew (RBG) website was also assessed for documentation of parameters like presence or absence of awns. Habitats of grasses were documented based of literature and field observations. As per compiled data, grass habitats were categorized in 9 categories (Tab. 1). Some grass species were exclusive to one habitat only, while some species were found to share two or more habitats.

Tab. 1. Total number of species per habitats associated with grassland and total number of species exclusive to habitat

\begin{tabular}{clcc}
\hline No. & Habitat & $\begin{array}{c}\text { Total } \\
\text { no. of } \\
\text { species }\end{array}$ & $\begin{array}{c}\text { Exclusive } \\
\text { species to } \\
\text { habitat }\end{array}$ \\
\hline 1 & Grassland grasses & 48 & 9 \\
2 & Weed in a crop field or Associated with & 36 & 5 \\
\hline 3 & crop fields & 4 & 3 \\
4 & Leashore or Costal regions & 1 & 0 \\
5 & Marshy areas & 62 & 17 \\
6 & Ruderal & 40 & 2 \\
7 & Undergrowth of forest & 31 & 9 \\
8 & Dry rocky areas & 20 & 8 \\
9 & Cultivated in field & 10 & 5 \\
\hline
\end{tabular}

Effort was made to provide palatability grades to grasses based on their utility values (Tab. 2). Highly palatable species are graded with $\mathrm{A}$, moderately palatable grasses are graded as $\mathrm{B}$, while the grass species which are consumed before the flowering and after the awn are dispersed are graded as $\mathrm{C}$. The species which are harvested and stored for their use at the time of non availability of grass or other fodder are also placed in grade $\mathrm{C}$.

Tab. 2. The palatability grades of grass species based on animal preferences

\begin{tabular}{ccl}
\hline No. & Grade & \multicolumn{1}{c}{ Palatability } \\
\hline 1 & A & Very good or excellent fodder \\
2 & B & $\begin{array}{l}\text { Good fodder grass } \\
\text { Grasses consumed when they are young or } \\
\text { consumed when good palatable fodder species are } \\
\text { not available }\end{array}$ \\
\hline & C & \\
\hline
\end{tabular}

Botanical names are updated as per online database available at The Plant List (2013). The names of the grass species in the table are arranged alphabetically for the sake of convenience. The references to the literature are also provided in the list.

\section{Results and discussion}

A total of 143 grasses from peninsular Indian region are recorded as a palatable, out of which 64 are awned and 79 are unawned (Tab. 3). The awned grasses are palatable only either before maturity of awns or after they fall off. All stages of unawned grasses are consumed by grazing animals. This can be one of the reasons for awned palatable grasses to be in lower number (64) when compared to unawned palatable grasses (79).

Nine types of habitats were categorized where palatable grass species seen to grow (Tab. 1). A total of 58 palatable grasses are exclusive to a single habitat, while the remaining 85 grasses share 2 or more habitats. The classification of grasses 
in these nine habitats revealed 17 palatable grasses exclusive to found on lateritic plateaus. Based on palatability potential, marshy habitat and only 3 palatable grasses were exclusively reported from the costal habitat. The highest number of palatable grasses was found in marshy habitat, which may be attributed to water availability throughout the year, while less number of grasses grows in costal habitat because of high salinity. The minimum number (1) of palatable species was grasses are provided with three artificial grades. Out of 143 grasses 22 are A grade, 72 are B grade and 49 belong to C grade. Details about these grades are given in Tab. 2. Amongst grade C grass species like Apluda muticas Sehima sp., Dichanthium sp., Isielema sp. etc. are cut, dried and stored for use as a fodder in summer season.

Tab. 3. Checklist of palatable grass species from Peninsular India with details like Presence or Absence of awns, Floral phenology, Vernacular names, Habitat, Palatability grade, References and Botanical names as per recent nomenclature

\begin{tabular}{|c|c|c|c|c|c|c|c|c|}
\hline No. & Name of Grasses & Awns & $\begin{array}{c}\text { Floral } \\
\text { Phenology }\end{array}$ & Vernacular names & Habitats & $\begin{array}{c}\text { Status of } \\
\text { occurrence }\end{array}$ & $\begin{array}{c}\text { Palatability } \\
\text { Grade\# }\end{array}$ & $\operatorname{Re}^{*}$ \\
\hline 1 & $\begin{array}{l}\text { Acrachne racemosa (B. Heyne. ex } \\
\text { Roth.) Ohwi. }\end{array}$ & $\mathrm{P}$ & Jul- Dec & Chinkhe & 1,5 & Rare & $\mathrm{C}$ & 1 \\
\hline 2 & Aeluropus lagopoides (L.) Thwaites. & A & Nov-Jan & Dolagavat & 3 & Occasional & B & 4 \\
\hline 3 & Alloteropsis cimicina Stafp. & $\mathrm{P}$ & Jul- Nov & Sinri & 1,9 & Common & B & $\begin{array}{c}2,3 \\
4\end{array}$ \\
\hline 4 & Andropogon fastigiatus $\mathrm{Sw}$. & $\mathrm{P}$ & Sep-Nov & & 8 & Occasional & A & 4 \\
\hline 5 & Andropogon pumilus Roxb. & $\mathrm{P}$ & Aug-Jan & $\begin{array}{c}\text { Baerki, Divartan, Gondval, lalgavat, } \\
\text { Tambadgota. }\end{array}$ & 8 & Frequent & A & $\begin{array}{c}2,4 \\
5\end{array}$ \\
\hline 6 & Apluda mutica L. & $\mathrm{P}$ & Oct- May & $\begin{array}{c}\text { Ghagara, Holera, Kharvel, } \\
\text { Phulia,Tambat, Makka, Tulsi, } \\
\text { poadi. }\end{array}$ & 1,7 & Frequent & B & $\begin{array}{c}3,4 \\
5\end{array}$ \\
\hline 7 & Aristida adscensionis L. & $\mathrm{P}$ & Feb- Apr & Longi-kasal & 6,9 & Common & $\mathrm{C}$ & 1,2 \\
\hline 8 & Aristida funiculata Trin. \& Rupr. & $\mathrm{P}$ & Sep-Dec & Bhuti, Pandharikusal & 1 & Common & $\mathrm{C}$ & 2 \\
\hline 9 & Aristida stocksii (Hook.f.) Domin. & $\mathrm{P}$ & Sep-Nov & & 6,8 & Rare & $\mathrm{C}$ & 2 \\
\hline 10 & Arthraxon lancefolious (Trin.) Hochst. & $\mathrm{P}$ & Aug-Dec & Faradyachne gavat, Turda & 1,8 & Common & B & 1 \\
\hline 11 & Arundinella leptochloa (Steud.) Hook. $f$. & A & Oct-Jun & & 7 & Occasional & B & 4 \\
\hline 12 & Arundinella pumilla (Hochst.) Steud. & $\mathrm{P}$ & Sep- Nov & & 7 & Common & $\mathrm{C}$ & 1 \\
\hline 13 & Bothriochloa bladhii (Retz.) S. T. Black & $\mathrm{P}$ & Sep-Jan & & 5 & & B & 2,3 \\
\hline 14 & Bothriochloa pertusa (L.) A. Camus & $\mathrm{P}$ & Oct- Dec & Ghanda, Kathora, Palva. & $1,6,2,5$ & Common & B & $\begin{array}{c}1,2 \\
4\end{array}$ \\
\hline 15 & Brachiaria distachya (L.) Stapf. & A & Aug-Dec & Motia & 1,7 & Occasional & $\mathrm{C}$ & 1,4 \\
\hline 16 & Brachiaria erusiformis (Sm.) Griseb. & A & Sep-Jan & Shimpi, Wag-hast & 2 & $\begin{array}{l}\text { Common } \\
\text { weed }\end{array}$ & $\mathrm{C}$ & $\begin{array}{c}1,2 \\
3\end{array}$ \\
\hline 17 & Brachiaria mutica (Forssk.) Stapf. & A & Aug-Jan & watergrass, Buffalow grass & 1 & Cultivate & A & 2,3 \\
\hline 18 & Brachiaria ramosa (L.) Stapf. & A & Aug- Nov & Chapar, chapsura & 1,5 & Common & B & 1,4 \\
\hline 19 & $\begin{array}{l}\text { Brachiaria reptans (L.) C. A. Gardner } \\
\text { \& C.E. Hubb. }\end{array}$ & A & Sep- Oct & Chimanchara, Chopli & 2,7 & Common & B & 3 \\
\hline 20 & Capilipedium assimile (Steud.) A. Camus & $\mathrm{P}$ & Sep- Dec & & 6,7 & Occasional & $\mathrm{C}$ & 3,4 \\
\hline 21 & Cenchrus biflorus Roxb. & A & Oct- Dec & Bhoront, Kukar & 3 & $\begin{array}{l}\text { Occasional } \\
\text { at shore }\end{array}$ & $\mathrm{C}$ & 1,5 \\
\hline 22 & Cenchrus ciliaris $\mathrm{L}$. & A & Jul- Dec & Anjandhaman & $6,8,9$ & $\begin{array}{l}\text { Occasional } \\
\text { in fields }\end{array}$ & A & $\begin{array}{c}1,3 \\
4\end{array}$ \\
\hline 23 & Cenchrus pennisetiformis Steud. & A & Aug-Sep & Sankaveth & 6,8 & & A & 3,4 \\
\hline 24 & $\begin{array}{l}\text { Chionachne gigantea (J. Koenig) } \\
\text { Veldkamp }\end{array}$ & A & Sep-Dec & Kanta-karvel, kasali, Varival & 5,6 & & $\mathrm{C}$ & 1,3 \\
\hline 25 & $\begin{array}{l}\text { Chionachne semiteres (Benth.) } \\
\text { Henrard. }\end{array}$ & A & Sep-Dec & & 2 & Rare & B & 4 \\
\hline 26 & Chloris barbata Sw. & $\mathrm{P}$ & Aug- Dec & Gondvel, Ghosha, Mesi & 2,6 & $\begin{array}{l}\text { Occasional } \\
\text { at wetlands }\end{array}$ & $\mathrm{C}$ & 3,5 \\
\hline 27 & Chloris bournei Rang. \& Tadul. & $\mathrm{P}$ & Sep-Jan & & 2,6 & $\begin{array}{l}\text { Occasional } \\
\text { at wetlands }\end{array}$ & $\mathrm{C}$ & 3 \\
\hline 28 & Chloris gayana Kunth. & $\mathrm{P}$ & Jul- Oct & & 8 & Occasional & A & 3,4 \\
\hline 29 & Chloris montana Roxb. & $\mathrm{P}$ & Sep- Oct & & 8 & Occasional & $\mathrm{C}$ & 2 \\
\hline 30 & Chloris virgata Swz. & $\mathrm{P}$ & Sep-Dec & Gholshep, Ghorapuchbi & 2,6 & $\begin{array}{l}\text { Common } \\
\text { on walls }\end{array}$ & B & 2,3 \\
\hline 31 & Chrysopogon aciculatus (Retz.) Trin. & $\mathrm{P}$ & Aug- Oct & & 6,5 & Occasional & $\mathrm{C}$ & 1 \\
\hline 32 & Chrysopogon fulvus (Spreng) Chiov. & $\mathrm{P}$ & Sep-Dec & Ariva, Gogar, Kahandol,Vagnakbi & 7 & Common & $\mathrm{C}$ & $\begin{array}{c}1,2 \\
3\end{array}$ \\
\hline 33 & Chrysopogon lancearius (Hook.f.) Haines. & $\mathrm{P}$ & Oct- Nov & & 1,4 & Rare & B & 1,3 \\
\hline 34 & $\begin{array}{l}\text { Chrysopogon orientalis (Desv.) A. } \\
\text { Camus. }\end{array}$ & $\mathrm{P}$ & Sep-Oct & & 1 & Rare & $\mathrm{C}$ & 3,4 \\
\hline
\end{tabular}




\begin{tabular}{|c|c|c|c|c|c|c|c|c|}
\hline 35 & $\begin{array}{l}\text { Chrysopogon polyphyllus(Hack.) Blatt. } \\
\text { \& McCann. }\end{array}$ & $\mathrm{P}$ & Sep- Dec & & 5,7 & Frequent & $\mathrm{B}$ & 3,4 \\
\hline 36 & Chrysopogon serrulatus Trin. & $\mathrm{P}$ & Jul- Dec & & 8,9 & Rare & $\mathrm{B}$ & 3,4 \\
\hline 37 & Coix aquatica Roxb. & A & Sep-Nov & Kachor & 5 & $\begin{array}{l}\text { Common along } \\
\text { streams }\end{array}$ & $\mathrm{B}$ & 3 \\
\hline 38 & Coix lacryma-jobi $\mathrm{L}$. & A & Aug-Jan & $\begin{array}{l}\text { Kasai, Kochura, randavar, Ran- } \\
\text { jondhala, Ran-mabai,Ran-maka }\end{array}$ & 5 & Rare & $\mathrm{C}$ & 3 \\
\hline 39 & Cynodon dactylon (L.) Pers. & A & $\begin{array}{l}\text { Throughout } \\
\text { the year. }\end{array}$ & Durva, Harali & 5,6 & Common & $\mathrm{B}$ & $\begin{array}{c}1,3,4 \\
5\end{array}$ \\
\hline 40 & Cynodon radiatus Roth. & A & Aug- Nov & Harali & 7 & $\begin{array}{l}\text { Common along } \\
\text { forest }\end{array}$ & $\mathrm{B}$ & 4 \\
\hline 41 & Dactyloctenium aegipticum (L.) Willd. & A & Aug-Feb & Gandhi, Anchi, Manchi, & 6,2 & Common & $\mathrm{C}$ & 2 \\
\hline 42 & Dactyloctenium aristatum Link. & A & Jul- Oct & & $1,2,6$ & $\begin{array}{l}\text { Common on } \\
\text { hill slops }\end{array}$ & B & 4 \\
\hline 43 & Dactyloctenium scindicum Boiss. & A & Sep-Nov & Bhara, Bobaria & 5 & Rare & $\mathrm{B}$ & 1 \\
\hline 44 & $\begin{array}{l}\text { Dichanthium annulatum (Forssk) } \\
\text { Stapf }\end{array}$ & $\mathrm{P}$ & Oct-Mar & Jinjiva, Marvel, Paunava & 1,6 & Common & A & 3,5 \\
\hline 45 & $\begin{array}{l}\text { Dichanthium armatum (Hook.f.) } \\
\text { Blatt. \& McCann. }\end{array}$ & $\mathrm{P}$ & Sep-Oct & & 7,8 & & $\mathrm{~B}$ & 4 \\
\hline 46 & $\begin{array}{l}\text { Dichanthium caricosum (L.) A. } \\
\text { Camus. }\end{array}$ & $\mathrm{P}$ & Sep-Dec & Jetare, Marvel & 1,6 & Frequent & A & 2,3 \\
\hline 47 & $\begin{array}{l}\text { Dichanthium foveolatum (Delile.) } \\
\text { Roberty. }\end{array}$ & $\mathrm{P}$ & Sep-Dec & Ghandel, Marvel & 1 & Common & $\mathrm{B}$ & 2 \\
\hline 48 & $\begin{array}{l}\text { Digitaria abludens (Roem. \& Schult.) } \\
\text { Veldkamp }\end{array}$ & A & Aug-Oct & & 1,5 & Occasional & $\mathrm{B}$ & 4 \\
\hline 49 & $\begin{array}{l}\text { Digitaria bicornis (Lam.) Roem \& } \\
\text { Shult. }\end{array}$ & A & Aug-Dec & & 2,7 & Common & $\mathrm{C}$ & 3,4 \\
\hline 50 & Digitaria ciliaris (Retz.) Koeler. & A & Aug- Feb & & 2,7 & Common & $\mathrm{B}$ & $\begin{array}{c}1,2,3 \\
4\end{array}$ \\
\hline 51 & Digitaria longiflora (Retz.) Pers. & A & Aug-Dec & & 1,6 & Frequent & $\mathrm{B}$ & $2,3,4$ \\
\hline 52 & Digitaria stricta Roth. & A & Aug- Feb & & 1,5 & Rare & $\mathrm{C}$ & 2,3 \\
\hline 53 & Dinebra retroflexa (Vahl) Panz. & A & Sep- Feb & Lonigavat,Lona, Kali kauli & 7 & Common weed & $\mathrm{B}$ & 2,4 \\
\hline 54 & Echinocloa colona (L.) Link. & A & Jul- Feb & $\begin{array}{l}\text { Borad, Bovur, Harund, Ranborat, } \\
\text { Rovar, Sama, Savank. }\end{array}$ & 2,5 & Common & $\mathrm{B}$ & $\begin{array}{c}1,2,4, \\
5\end{array}$ \\
\hline 55 & Eleusine indica (Linn.) Geart. & A & Sep- Feb & Mahar-nachani, Mendala, Mela & $1,5,6$ & Common & $\mathrm{B}$ & $3,4,5$ \\
\hline 56 & $\begin{array}{l}\text { Enteropogon dolichostachya (Lag.) } \\
\text { Keng }\end{array}$ & $\mathrm{P}$ & Sep-Dec & & 7 & & $\mathrm{C}$ & 2 \\
\hline 57 & Eragrostis amabilis (L.) Wight \& Arn. & A & Sep-Mar & Bakralu, Belakuda, Waya, Dhani. & 6 & & $\mathrm{~B}$ & 1 \\
\hline 58 & Eragrostis aspera (Jacq.) Nees & A & Oct- Dec & & 2 & $\begin{array}{l}\text { Occasional } \\
\text { weed }\end{array}$ & $\mathrm{C}$ & 2,3 \\
\hline 59 & Eragrostis cilianensis (All.) Janch & A & Aug-May & Ranpohe, Pohe & 1 & Occasional & $\mathrm{C}$ & 3 \\
\hline 60 & Eragrostis ciliaris (L.) R. Br. & A & Sep-Jan & $\begin{array}{c}\text { Undir-panjo, Tor chandbol, } \\
\text { Burbudi. }\end{array}$ & 5 & Common & $\mathrm{B}$ & 1,4 \\
\hline 61 & Eragrostis gangetica (Roxb.) Steud. & A & Jul-Mar & Todha, Asara, chota asara, Kalaurgi & 5 & Occasional & B & 1,4 \\
\hline 62 & Eragrostisjaponica (Thunb.) Trin. & A & Sep-Mar & Chiksi, Dhuria, Shetpatra & 2,5 & Occasional & $\mathrm{B}$ & 2,4 \\
\hline 63 & $\begin{array}{l}\text { Eragrostis nutans (Retz.) Nees ex. } \\
\text { Steud. }\end{array}$ & A & Oct-Dec & Fulia, chikta, Khari & 5 & $\begin{array}{l}\text { Common at } \\
\text { wet places }\end{array}$ & $\mathrm{B}$ & 1 \\
\hline 64 & Eragrostispilosa (L.) P. Beauv. & A & Jul-Oct & Burwai, chiriaka dana, Kutki & 5,6 & Common & $\mathrm{B}$ & 1,4 \\
\hline 65 & Eragrostis patula (Kunth) Steud. & A & Sep-Oct & & 5,6 & $\begin{array}{l}\text { Common on } \\
\text { wet soil }\end{array}$ & $\mathrm{B}$ & 2 \\
\hline 66 & $\begin{array}{l}\text { Eragrostis unioloids (Retz.) Nees Ex } \\
\text { steud. }\end{array}$ & A & Aug- Feb & Chidsi, Chimandara, Holpoho & $2,5,6$ & Frequent & $\mathrm{B}$ & 1 \\
\hline 67 & Eragrostis viscosa (Retz.) Trin. & A & Jul- Dec & Bhubhur, Bhulmi, Chilpal, Chitki & 2,6 & Frequent & $\mathrm{C}$ & 1 \\
\hline 68 & Eriochloa procera (Retz.) C.E. Hubb. & A & Oct-Jan & & 5 & $\begin{array}{l}\text { Occasional to } \\
\text { wet places }\end{array}$ & $\mathrm{C}$ & $1,2,4$ \\
\hline 69 & Eulalia trispicata (Shult.) Henr. & $\mathrm{P}$ & Oct- Dec & & 1,7 & $\begin{array}{l}\text { Frequent to } \\
\text { grassland }\end{array}$ & C & $1,3,4$ \\
\hline 70 & Hackelochloa granularis (L.) Kuntze & A & Aug- Oct & Dalura-ghas, Kanjani, Kangri & 1,7 & Common & $\mathrm{B}$ & 3,5 \\
\hline 71 & Hemarthria compressa (L.f.) R. Br. & A & Nov-Dec & Baika & 5 & Rare & $\mathrm{B}$ & $\begin{array}{c}1,2,3, \\
4\end{array}$ \\
\hline 72 & $\begin{array}{l}\text { Heteropogon contortus (L.) P. Beauv. ex } \\
\text { Roem \& Schult }\end{array}$ & $\mathrm{P}$ & Aug- Feb & Kali-kusali, Kursali, Nanisunkhali & $1,5,8$ & Common & $\mathrm{C}$ & 3,4 \\
\hline 73 & $\begin{array}{l}\text { Heteropogon ritchiei (Hook.f.) Blatt. \& } \\
\text { McCann. }\end{array}$ & $\mathrm{P}$ & Aug- Nov & & 5,7 & Occasional & B & \\
\hline
\end{tabular}




\begin{tabular}{|c|c|c|c|c|c|c|c|c|}
\hline 74 & $\begin{array}{l}\text { Hygrorhyza aristata (Retz.) Nees. ex } \\
\text { Wight \& Arn }\end{array}$ & $\mathrm{P}$ & Nov-Mar & Deobhat, Urodhan & 5 & Occasional & $\mathrm{B}$ & $\begin{array}{c}1,3 \\
4\end{array}$ \\
\hline 75 & Imperata cyclindrica (L.) Raeusch. & A & Aug-Nov & Dhup & 5 & Occasional & $\mathrm{C}$ & 2,3 \\
\hline 76 & Isachne globosa (Thunb.) Kuntze. & A & Aug- Feb & Daura & 2,5 & $\begin{array}{l}\text { Frequent to } \\
\text { marshy places }\end{array}$ & $\mathrm{B}$ & $\begin{array}{c}1,3 \\
4\end{array}$ \\
\hline 77 & Isachne meeboldii C.E.C. Fisch. & $\mathrm{A}$ & Aug-Dec & & $2,5,6$ & $\begin{array}{l}\text { Occasional in } \\
\text { grasslands }\end{array}$ & $\mathrm{B}$ & 2 \\
\hline 78 & $\begin{array}{l}\text { Ischaemum afrum (J. F. Glem) } \\
\text { Dandy }\end{array}$ & $\mathrm{P}$ & Sep-Jan & Kunda, Nuth & 2 & & A & 2 \\
\hline 79 & Ischaemum timorense Kunth. & $\mathrm{P}$ & Oct- Nov & & $5,6,7$ & Occasional & $\mathrm{C}$ & 3,4 \\
\hline 80 & Ischeamum semisagittatum Roxb. & $\mathrm{P}$ & Oct-Dec & Ber, Dalaga & 6,7 & Occasional & $\mathrm{B}$ & 4 \\
\hline 81 & Iseilema anthephoroides Hack. & $\mathrm{P}$ & Aug-Dec & Tambadgota & $2,5,6$ & Frequent & A & $\begin{array}{c}2,3 \\
5\end{array}$ \\
\hline 82 & Iseilema holei Haines. & $\mathrm{P}$ & Oct- Feb & & 7 & Rare & $\mathrm{B}$ & 2 \\
\hline 83 & Iseilema laxum Hack. & $\mathrm{p}$ & Aug-Dec & Moshi, Mus, Shadta,Tambit. & 1,5 & Occasional & A & $\begin{array}{c}2,3 \\
4\end{array}$ \\
\hline 84 & Iseilema prostratum (L.) Andersson. & $\mathrm{P}$ & Oct-Jan & Achigrass, Sona & 1,5 & Common & $\mathrm{B}$ & 1 \\
\hline 85 & Leersia hexandra Sw. & A & $\begin{array}{c}\text { Throughout } \\
\text { the year }\end{array}$ & & 5 & Occasional & $\mathrm{B}$ & 1,4 \\
\hline 86 & Leptochloa chinensis (L.) Nees & A & Sep- Feb & Chenhel, Jhira, Phulkia & 2,5 & Rare & $\mathrm{C}$ & 2 \\
\hline 87 & Leptochloa fusca (L.) Kuntz. & $\mathrm{P}$ & Sep- Oct & & 3,5 & & $\mathrm{C}$ & 1 \\
\hline 88 & $\begin{array}{l}\text { Lophopogon tridentatus (Roxb.) } \\
\text { Hack. }\end{array}$ & $\mathrm{P}$ & Aug- Dec & & 1,5 & Common & $\mathrm{B}$ & 4 \\
\hline 89 & $\begin{array}{l}\text { Melanocenchris abyssinica (R. Br. ex } \\
\text { Fresen.) Hochst. }\end{array}$ & $\mathrm{P}$ & Aug-Sep & & 1,8 & & $\mathrm{C}$ & 1 \\
\hline 90 & Ochthochloa compressa (Forssk.) Hilu & A & Jul- Nov & & 8 & & $\mathrm{~B}$ & 1,4 \\
\hline 91 & Ophiuros exaltatus (L.) Ketze. & A & Sep-Dec & Hutia, Uphada & 2,6 & Occasional & $\mathrm{B}$ & 4 \\
\hline 92 & $\begin{array}{l}\text { Oplismenus burmanni (Retz.) P. } \\
\text { Beauv. }\end{array}$ & A & Jul- Feb & Kudak, Yerwa & 2,7 & $\begin{array}{l}\text { Common to shady } \\
\text { places }\end{array}$ & $\mathrm{B}$ & 2,4 \\
\hline 93 & Oplismenus compositus (L.) P. Beauv. & A & Aug-Dec & Shara, Turdia & 5,7 & $\begin{array}{l}\text { Common to shady } \\
\text { places }\end{array}$ & $\mathrm{B}$ & 4 \\
\hline 94 & Oryza rufipogon Griff. & $\mathrm{P}$ & Sep-Dec & & 5 & Occasional & C & 2,3 \\
\hline 95 & $\begin{array}{l}\text { Panicum atrosanguineum Hochst. ex } \\
\text { A. Rich. }\end{array}$ & $\mathrm{A}$ & Oct- Dec & & 7 & & $\mathrm{~A}$ & 4 \\
\hline 96 & Panicum curviflorum Hornem & A & Oct- Nov & Bhatur & 1 & & A & 3 \\
\hline 97 & Panicum miliaceum L. & A & Oct-Dec & Ghoti sava, varai, Chinee & 9 & Cultivate & A & 1,3 \\
\hline 98 & Panicum paludosum Roxb. & A & Aug- Feb & Borati, Kulus-nan & 2,5 & Occasional & $\mathrm{B}$ & 2,3 \\
\hline 99 & Panicum repens $\mathrm{L}$. & A & Oct- Nov & & 2,5 & Occasional & $\mathrm{B}$ & $\begin{array}{c}2,4, \\
5\end{array}$ \\
\hline 100 & Panicum sumatrense Roth. & A & Sep-Nov & Sava & 9 & Occasional & $\mathrm{B}$ & 1,4 \\
\hline 101 & $\begin{array}{l}\text { Paspalidium flavidum (Retz.) A. } \\
\text { Camus. }\end{array}$ & $\mathrm{A}$ & Jul-Oct & Burad, Shedya & 5,7 & Frequent & $\mathrm{B}$ & 2,4 \\
\hline 102 & $\begin{array}{l}\text { Paspalidium geminatum (Forssk.) } \\
\text { Stapf. }\end{array}$ & A & Sep-Mar & & 5 & Common & $\mathrm{B}$ & 3,4 \\
\hline 103 & $\begin{array}{l}\text { Paspalidium punctatum (Burm.) A. } \\
\text { Camus. }\end{array}$ & A & Sep-Dec & Petnar, Dossa & 5 & & $\mathrm{C}$ & 4 \\
\hline 104 & Paspalum dilatatum Poir. & A & Jun- Aug & & $5,6,9$ & Rare & A & $\begin{array}{c}1,3 \\
4\end{array}$ \\
\hline 105 & Paspalum scrobiculatum L. & A & Aug-Apr & Harik, Kodra, Majore, Pakodi & 2,5 & Frequent & $\mathrm{B}$ & 3,4 \\
\hline 106 & $\begin{array}{l}\text { Pennisetum hohenackerii Hochst. ex. } \\
\text { Steud. }\end{array}$ & A & Aug- Feb & Mobl & 2,5 & Frequent & A & 2 \\
\hline 107 & Pennisetum pedicellatum Trin. & A & Aug-Jan & & 1,6 & Frequent & A & 3,4 \\
\hline 108 & Pennisetum purpureum Schumach. & A & Oct- Dec & Elephant grass & 9 & Cultivate & $\mathrm{C}$ & $\begin{array}{c}1,4, \\
3\end{array}$ \\
\hline 109 & Perotis indica (L.) Kuntze. & A & Oct- Aug & Kurad, Khuras & 1,8 & Occasional & $\mathrm{B}$ & 4 \\
\hline 110 & Polypogon monspeliensis (L.) Desf. & $\mathrm{P}$ & Jul- Feb & & 2,5 & Rare & A & 1,3 \\
\hline 111 & $\begin{array}{l}\text { Psudanthistiria heteroclita (Roxb.) } \\
\text { Hook.f. }\end{array}$ & $\mathrm{P}$ & Sep-Jan & Pokalya & 1,7 & $\begin{array}{l}\text { Frequent in } \\
\text { grasslands }\end{array}$ & B & 2,4 \\
\hline 112 & $\begin{array}{l}\text { Rottboellia cochinchinensis (Lour.) } \\
\text { Clayton. }\end{array}$ & A & Sep-Dec & Bura & 2,5 & Occasional & B & 4 \\
\hline 113 & Saccharum ravennae (L.) L. & $\mathrm{P}$ & Dec & & 8 & & $\mathrm{C}$ & 1,3 \\
\hline 114 & Saccharum spontaneum L. & A & Sep- Dec & $\begin{array}{c}\text { Bagheri, Kamis, Khair, Bochari, } \\
\text { Kan }\end{array}$ & 5 & Occasional & $\mathrm{C}$ & 2,4 \\
\hline 115 & Sacciolepis indica (L.) Chase. & A & Sep- Dec & & 2,5 & Common in & $\mathrm{B}$ & 4 \\
\hline
\end{tabular}




\begin{tabular}{|c|c|c|c|c|c|c|c|c|}
\hline 116 & Sacciolepis interrupta (Willd.) Stapf. & A & Oct- Dec & Pokalia & 5 & $\begin{array}{l}\text { Occasional in } \\
\text { wetlands }\end{array}$ & B & 2,3 \\
\hline 117 & $\begin{array}{l}\text { Sacciolepis myosuroides (R. Br.) A. } \\
\text { Camus. }\end{array}$ & A & Nov- Feb & Kura-lom, Pokalia, Didhina & 2,5 & $\begin{array}{l}\text { Occasional in rice } \\
\text { fields }\end{array}$ & B & 4 \\
\hline 118 & Schizachyrium exile (Hochst.) Pilg. & $\mathrm{P}$ & Aug- Nov & & 1,5 & Rare & $\mathrm{C}$ & 3,4 \\
\hline 119 & Sehima ischaemoides Forssk. & $\mathrm{P}$ & Sep- Dec & Paunat, Sheda & 1 & Rare & B & 3,4 \\
\hline 120 & Sehima nervosum (Rottl.) Stapf. & $\mathrm{P}$ & Sep-Oct & Pavnat, Paunya, Pavna, Sheda & 1 & Common & A & $\begin{array}{c}2,4 \\
5\end{array}$ \\
\hline 121 & Sehima notatum (Hack.) A. Camus. & $\mathrm{P}$ & Aug- Dec & Pavana & 1,7 & $\begin{array}{l}\text { Frequent in opne } \\
\text { grasslads }\end{array}$ & B & 2 \\
\hline 122 & $\begin{array}{l}\text { Sehima sulcatum (Hack.) A. } \\
\text { Camus. }\end{array}$ & $P$ & Aug-Nov & Pavana, Sheda & 1,2 & Common & A & 2,5 \\
\hline 123 & Setaria intermedia Roem. \& Schult. & A & Aug-Jan & Lundi, Landgar, Pandar, sava & 5,7 & $\begin{array}{l}\text { Frequent in } \\
\text { shades }\end{array}$ & $\mathrm{C}$ & 2 \\
\hline 124 & $\begin{array}{l}\text { Setaria pumila (Poir.) Roem. \& } \\
\text { Schult. }\end{array}$ & A & Jul- Nov & Bindi, Kolara, Kolu, Kolwa & $1,5,6$ & Common & B & $\begin{array}{c}2,3 \\
5\end{array}$ \\
\hline 125 & Setaria verticillata (L.) P. Beauv. & A & Sep-Jan & Danyani, Lapti, Bardani, chilaya & 6 & $\begin{array}{l}\text { Occasional to } \\
\text { shady places }\end{array}$ & $\mathrm{C}$ & 1,5 \\
\hline 126 & $\begin{array}{l}\text { Sorghum controversum (Steud.) } \\
\text { Snowden. }\end{array}$ & $\mathrm{P}$ & Sep-Jan & & 2,5 & $\begin{array}{l}\text { Occasional in } \\
\text { bunds }\end{array}$ & B & 2 \\
\hline 127 & $\begin{array}{l}\text { Sorghum deccanens Stapf. ex } \\
\text { Raizada. }\end{array}$ & $\mathrm{P}$ & Sep- Dec & Kakla & 2 & $\begin{array}{l}\text { Common along } \\
\text { bunds }\end{array}$ & B & 2 \\
\hline 128 & $\begin{array}{l}\text { Sporobolus coromandelianus (Retz.) } \\
\text { Kunth. }\end{array}$ & A & Nov- Jan & & 1 & $\begin{array}{l}\text { Frequent in } \\
\text { grasslands }\end{array}$ & A & 2 \\
\hline 129 & $\begin{array}{l}\text { Sporobolus helvolus (Trin.) T. } \\
\text { Durand. \& Schinz }\end{array}$ & A & Nov- Dec & & 8 & & B & 3,4 \\
\hline 130 & Sporobolus indicus (L.) R. Br. & A & Jul- Nov & Ghorla & 1,6 & Common & A & 2,5 \\
\hline 131 & Sporobolus maderaspatanus Bor. & A & Aug-Dec & & 3 & & $\mathrm{~B}$ & 1,3 \\
\hline 132 & Tetrapogon tenellus (Roxb.) Chiov. & $P$ & Aug-Dec & & 1,6 & $\begin{array}{l}\text { Occasional in } \\
\text { grasslands }\end{array}$ & B & $\begin{array}{c}1,3 \\
4\end{array}$ \\
\hline 133 & Thelepogon elegans Roth. & $\mathrm{P}$ & Sep-Jan & Kharbadi, Tirpha, Kadi & $1,2,6$ & $\begin{array}{l}\text { Frequent in } \\
\text { grasslands }\end{array}$ & $\mathrm{C}$ & 3,4 \\
\hline 134 & Themeda cymbaria Hack. & $\mathrm{P}$ & Aug- Oct & Karar, Fulgavat & 7 & & $\mathrm{C}$ & $\begin{array}{c}1,3 \\
4\end{array}$ \\
\hline 135 & Themeda quadrivalis (L.) Ktze. & $\mathrm{P}$ & Sep-Jan & Bhati, Fulora, Zini, Bhataru & 1,6 & Common & $\mathrm{C}$ & $\begin{array}{l}2,3 \\
4,5\end{array}$ \\
\hline 136 & $\begin{array}{l}\text { Themeda tremula (Nees. ex stued.) } \\
\text { Hack. }\end{array}$ & $\mathrm{P}$ & Oct- Feb & Barki, Bhatandi, Gundi & $1,6,7$ & Common & $\mathrm{C}$ & $\begin{array}{c}1,3 \\
4\end{array}$ \\
\hline 137 & Themeda triandra Forssk. & $\mathrm{P}$ & Sep- Feb & Batani,Bunden, Murar, Bhoru & 5,7 & $\begin{array}{l}\text { Frequent along } \\
\text { streams }\end{array}$ & $\mathrm{C}$ & 2,3 \\
\hline 138 & Tragus mongolorum Ohwi & A & Aug-Sep & & 1,8 & & $\mathrm{C}$ & 1 \\
\hline 139 & Tricholaena teneriffae (L.f.) Link. & A & Sep- Dec & & 8 & Rare & $\mathrm{C}$ & 3,4 \\
\hline 140 & Tripogon bromoides Roth. & $\mathrm{P}$ & Jul- Dec & & $1,7,8$ & Occasional & $\mathrm{C}$ & 2 \\
\hline 141 & Tripogon jaquemontii Stapf. & $\mathrm{P}$ & Aug-Nov & & $1,6,8$ & Common & $\mathrm{C}$ & 2 \\
\hline 142 & Urochloa panicoides P. Beauv. & $\mathrm{P}$ & May-Sep & Padhya, Kuri, Kuriya & 1,2 & Common & B & 1,2 \\
\hline 143 & Urocbloa setigera (Retz.) Stapf. & $\mathrm{P}$ & Jul- Dec & & $2,5,6$ & Occasional to & $\mathrm{B}$ & 1 \\
\hline
\end{tabular}

Note: Abbreviations: Awns: P- Present; A- Absent. Habitats: 1- Grassland grass; 2- Weed in a crop fields or Associated with crop fields; 3- Sea shore; 4- Lateritic platues; 5- Marshy areas; 6- Ruderal; 7- Undergrowth of forests; 8- Dry rocky areas; 9- Cultivated. Status: Occurrence of the species. Palatability Grade: A-Very good or excellent fodder; B-Good fodder grass; C- Grasses consumed when they young or consumed when good palatable fodder species are not available. ${ }^{*}$ References: 1 - Blatter $\&$ McCann (1935); 2- Patunkar (1980); 3- Bor (1960); 4- Potdar (2012); 5- Kulkarni et al. (1995).

\section{Conclusion}

A checklist of palatable grass species from Peninsular India was compiled to understand the status of fodder potential of grasslands and other habitats. The grasslands are dominated by un-palatable species as these species are slowly replacing palatable species, due to anthropogenic pressures like burning and grazing. All palatable grass species show a different palatability grade which can be assessed based on their consumption and animal preference to eat them. Large numbers of palatable grasses seem to prefer marshy habitat while sea shores and lateritic plateaus shelter a minimum number of palatable species.

\section{References}

Blatter E, McCann C (1935). The Bombay Grasses. Imperial Council of Agricultural Research. Scientific Monograph (5):323.

Bor NL (1960). The Grasses of Burma, Ceylon, India and Pakistan. Pergamon Press Oxford, London, New York Paris. $767 \mathrm{p}$.

Champion HG, Seth SK (1968). A Revised Survey of the Forest Types of India. Gov. of India Press, Delhi.

Dabadghao PM (1951). The Place of Grasses and Grasslands in our National Economy. Science and Culture 17:233-237.

Dabadghao PM, Shankarnarayan KA (1973). The Grass 
Cover of India. ICAR, New Delhi, p. 711.

Gadgil MD, Malhotra KC (1982). Ecology of a pastoral caste: Gavli Dhangars of peninsular India. Human Ecol 10(1):107-143.

Gadgil MD (1993). Restoring the productivity of Indian savannas. Man Biosphere Series 12:221-221.

Clayton WD, Vorontsova MS, Harman KT, Williamson H (2006 onwards). Grass base - The Online World Grass Flora. http://www.kew.org/data/grasses.db.html (assessed 1 June 2014).

Heady FH (1964). Palatability of Herbage and Animal Preference. J Range Managem 76-82.

Hussain F, Durrani MJ (2009). Seasonal Availability, Palatability and Animal Preferences of Forage Plants In Harboi Arid Rangeland, Kalat, Pakistan. Pak J Bot 41(2):539-554.

Ivins JD (1952). The Relative Palatability of Herbage Plants J Br Grassl Soc 7:43-54.

Javed IS, Inam-Ur-Rahim, Haq N, Muhammad Y, Javed I (2008). Mineral Composition, Palatability and Digestibility of Free Rangeland Grasses of Southern Grasslands of Pakistan. Pak J Bot 40(5):2059-2070. http://www.pakbs.org/pjbot/PDFs/40(1)/PJB40(1)237.p df

Kulkarni DK, Kumbhojkar MS (1995). Palatable Fodder Grasses from Pachgaon Parvati Area in Pune District. J Econ Tax Bot 19(3):529-532.

Lakshminarsimhan P in Sharma DB, Karthikeyan S, Singh NP (eds) (1996). Flora of Maharashtra StateMonocotyledons. Botanical Survey of India, Calcutta. p. 794.
Mani MS (1974). Biogeography of Peninsula. Monographiae Bioligicae 614-647

Mirza SN, Muhammad N, Quamar IA (2002). Effect of Growth Stages on the Yield and Quality of Forage Grasses. Pakistan J Agric Res 17(2):145-147.

Moretto AS, Distel RA (1999). Effect of selective defoliation on competitive interaction between palatable and unpalatable grasses native to temperate semiarid grassland of Argentina. J Arid Environm 42(3):167-175.

Naik VN, Patunkar BV (1979). Synecological Studies in Grasslands of Marathwada. Ind J For 2(4):372-377.

Oke JG (1973). Studies in grassland community in Maharashtra State: ecological classification of grassland patterns found in different ecological habitats and their botanical characterization. Indian Forest 86-106.

Patunkar BW (1980). Grasses or Marathwada. Scientific publishers, Jodhpur, p. 300.

Pemadasa MA (1990). Grasslands of the World. J Biogeograp 17:395-400.

Potdar GG, Salunkhe CB, Yadav SR (2012). Grasses of Maharashtra. Shivaji University Kolhapur, p. 656.

Suttie JM, Reynolds SG, Batello C (2005). Grasslands of the World. Food and Agriculture Organization of the United Nations, Rome, p. 514.

The Plant List (2013). Version 1.11 Published on Internet; http://www.theplantlist.org/ (assessed 1 June 2014).

Young MD, Solbrig OT (1993). The world savannas economic driving forces, ecological constrains and policy option for suitable land use. Man and the Biosphere Series 12:350. 